\title{
Is Hashimoto thyroiditis associated with increasing risk of thyroid malignancies? A systematic review and meta-analysis
}

\author{
Pouya Abbasgholizadeh ${ }^{1}$, Amirreza Naseri ${ }^{1,2}$ (D) Ehsan Nasiri ${ }^{1,3}$ (i) and Vahideh Sadra ${ }^{4^{*}}$ (D)
}

\begin{abstract}
Background and purpose: Hashimoto thyroiditis $(H T)$ is the most common inflammatory autoimmune thyroid disease and also the most common cause of hypothyroidism in developed countries. There is evidence of the role of HT in developing thyroid cancers (TCs). This study investigated the association between HT and different types of TCs.

Methods: Results of a comprehensive search in three major databases, as well as hand searching, were screened in title/abstract and full-text stages and the relevant data were extracted from the studies that met the inclusion criteria. Risk of bias (RoB) was assessed using the Joanna Briggs Institute (JBI) critical appraisal tools and the meta-analysis was conducted with Comprehensive Meta-Analysis software.
\end{abstract}

Results: Out of 4785 records, 50 studies were included in the systematic review, and 27 of them met the criteria for quantitative synthesis. The results indicated a significant role for HT in developing papillary TC (OR: 1.65; 95\% Cl: 1.04 to 2.61), medullary TC (OR: 2.70; 95\% Cl: 1.20 to 6.07) and lymphoma (OR:12.92; 95\% Cl: 2.15 to 77.63 ); but not anaplastic TC (OR: $1.92 ; 95 \%$ Cl: 0.29 to 1.90) and follicular TC (OR: $0.73 ; 95 \%$ Cl: 0.41 to 1.27 ). Also, this study found a significant association between $\mathrm{HT}$ and thyroid malignancies (OR: 1.36; $95 \% \mathrm{Cl}: 1.05$ to 1.77).

Conclusion: Although we found a significant association between HT and some types of TCs, High RoB studies, high level of heterogeneity, and the limited number of well-designed prospective studies, suggested the need for more studies to reach more reliable evidence.

Keywords: Hashimoto Thyroiditis, Chronic autoimmune thyroiditis, Thyroid neoplasms, Systematic review, Metaanalysis

\section{Introduction}

Chronic lymphocytic thyroiditis also called "Hashimoto thyroiditis" (HT) is the most common inflammatory autoimmune thyroid disease and the most common cause of hypothyroidism in regions with adequate amounts of iodine [1]. HT was first delineated by Japanese surgeon Hakaru Hashimoto as an autoimmune disease [2]. HT is characterized by immune cells infiltration of the

*Correspondence: sadra.vahideh@gmail.com

${ }^{4}$ Endocrine Research Center, Tabriz University of Medical Sciences, Golgasht street, Tabriz, Iran

Full list of author information is available at the end of the article thyroid gland as a result of failure in immune tolerance. This condition frequently affects females (more than 10:1 ratio of females to males) [3]. The occurrence of HT has increased during the last decades. Thyroid cancer (TC) is the most common endocrine tumor and the occurrence of TC has increased rapidly worldwide. Papillary thyroid carcinoma (PTC) is the most common type of thyroid neoplasms and accounts for $80-90 \%$ of all thyroid cancers. It occurs more frequently in females.

Rudolf Virchow first described the link between chronic inflammation and cancer in 1893, which is now well determined [4]. The association between HT and PTC was first described by Dailey et al. in 1955 [5]. original author(s) and the source, provide a link to the Creative Commons licence, and indicate if changes were made. The images or other third party material in this article are included in the article's Creative Commons licence, unless indicated otherwise in a credit line to the material. If material is not included in the article's Creative Commons licence and your intended use is not permitted by statutory regulation or exceeds the permitted use, you will need to obtain permission directly from the copyright holder. To view a copy of this licence, visit http://creativecommons.org/licenses/by/4.0/. The Creative Commons Public Domain Dedication waiver (http://creativeco mmons.org/publicdomain/zero/1.0/) applies to the data made available in this article, unless otherwise stated in a credit line to the data. 
Despite several retrospective and prospective studies performed, the relationship between them remains controversial. A recent meta-analysis of 64,628 patients in 36 studies reported a relation between HT and PTC and an association between HT and thyroid lymphoma [6]. Consistently with this finding, several studies have been performed and they reported that HT is associated with a greater probability of developing PTC [7]. Another metaanalysis revealed the correlation between HT and PTC and this systematic review only investigated the incidence of HT in TC patients and not the incidence of TC in HT patients [8]. In contrast with this finding, Jankovic et al. reported no significant association between HT and TC based on 8 fine-needle aspiration studies [9].

Given the selection bias and limitations of previous studies as well as new publications in this area, an updated systematic review is needed to better clarify the association between HT and TC. Therefore, we elaborated a new meta-analysis via a complete investigation of the literature aiming to evaluate the association between HT and TCs, and also the investigation of the role of $\mathrm{HT}$ in different subgroups of TC, based on current knowledge.

\section{Methods}

This systematic review was conducted following the Preferred Reporting Items for Systematic Reviews and MetaAnalyses (PRISMA) statement [10].

\section{Search}

After getting the approval of the study protocol, an electronic search was conducted in 3 major databases including Medline via PubMed, EMBASE, and Scopus, with ((Chronic autoimmune thyroiditis) OR Hashimoto) AND (thyroid neoplasm* OR thyroid carcinoma* OR thyroid cancer* OR thyroid adenoma* OR thyroid malignanc*) and related MeSH keywords on 23 February 2021.

\section{Study selection}

Results of the electronic search were imported into EndNote 20 and after removing the duplicated studies, the remaining records were screened in two title/ abstract and full-text stages. Two independent authors screened the studies and in case of any disagreements, a third author deemed the issue. For full coverage of any published studies, after selecting the final articles to be included in this systematic review, the reference lists of these articles and recently published reviews have been checked for possible inclusion in our study.

\section{Eligibility criteria}

We included the journal articles which assess the possible relation between $\mathrm{HT}$ and TC with both retrospective and prospective study designs. In case of lack of a control group, the study was included in our systematic review but excluded from the meta-analysis. We only selected the articles which have been written in English and animal studies, case reports, review articles, editorials, letters, conference abstracts, and withdrawn articles were excluded from our study.

\section{Data extraction}

Data extraction was conducted by two authors with an electronic table in Microsoft Word. The following data were extracted from each study: the name of the first author of the study, the year of study publication, the study design which could be retrospective or prospective, the setting of the study, the method for diagnosis of thyroid cancer, the sample size, the mean and standard deviation of ages, the number of female and male cases, the type of thyroid cancer and finally the rate of TC between HT cases and control group, or rate of HT between TC and control group.

\section{Risk of Bias assessment}

The risk of bias (RoB) in included studies was assessed using the Joanna Briggs Institute (JBI) critical appraisal tools for cohort or case-control studies based on the study design [11]. The checklist for case-control studies includes 10 questions and the cohort studies' checklist includes 11 questions. These tools assess the similarity of case and control groups, using a standard and similar method for assessing the condition, appropriate dealing with cofounding factors, enough period of interest, and appropriate statistical analysis.

\section{Statistics}

All the statistical analyses were conducted using the second version of Comprehensive Meta-Analysis (CMA.2) software with $95 \%$ confidence intervals and a 0.05 level of significance. $\mathrm{I}^{2}$ model was used for assessing the heterogeneity between the studies, and for outcomes with more than $50 \%$ level of heterogeneity, a random effect model was used. The number of events in case and control groups in both study designs and also the size of the group was imported into the CMA and the odds ratio (OR) was collected for each study. Then the results were then combined in both random and fixed effect models and the ORs for each subgroup (based on the type of thyroid cancer) as well as the overall result were calculated and presented by forest plot.

\section{Results}

Globally, 7141 records were identified through database searching, and after removing the duplicated studies, 4785 studies were screened. Finally, 50 studies were 
selected for qualitative synthesis and 27 of them, were included in the quantitative synthesis (Fig. 1). Among them, 23 studies found the rate of HT in TC cases; on the other hand, 29 of them assessed the rate of TC in HT cases, whereas 3 of them reported both of these findings. The characteristics of the included studies are summarized in Table 1. Figure 2 summarized the results of the meta-analysis.

\section{Papillary thyroid cancer}

45 of the included studies investigated the possible relation between $\mathrm{HT}$ and PTC. In these studies, the rate of HT in PTC cases was ranged between 4.75 to $38.4 \%$, whereas the rate of PTC in HT ranged between 0.12 to $64.3 \%$. The meta-analysis of 23 studies with an appropriate control group, found 1.65 OR (95\% CI: 1.04 to 2.61 ; $\mathrm{I}^{2}$ test for heterogeneity: $96.48 \%$ ) and the difference between the groups was significant $(p=0.03)$.

\section{Follicular thyroid cancer}

13 studies assessed the possible association between HT and follicular thyroid cancer (FTC). In these studies, the rate of HT in the FTC group ranged between 2.08 to $9.62 \%$ and the rate of FTC in HT ranged between 0 to $9.2 \%$. The meta-analysis of the 7 studies that met the proper inclusion criteria, reached 0.73 OR (95\% CI: 0.41 to 1.27 ; $\mathrm{I}^{2}$ test for heterogeneity: $0 \%$ ) and the difference between the groups was not statistically significant $(p=0.26)$.

\section{Medullary thyroid cancer}

The possible role of HT in developing medullary thyroid cancer (MTC) was investigated in 6 studies. All of these studies assessed the rate of MTC in the HT group and it

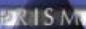

PRISMA 2009 Flow Diagram
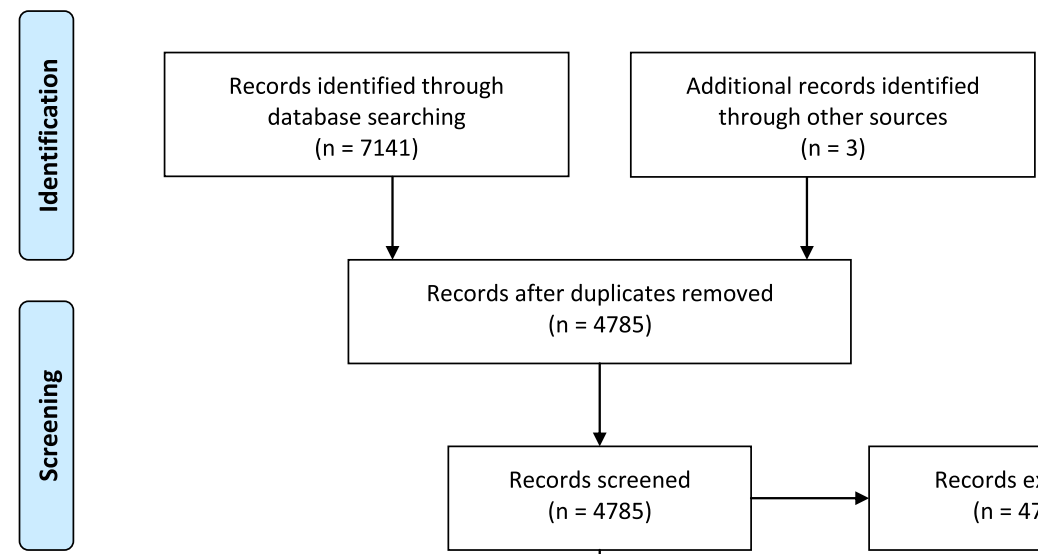

Records after duplicates removed ( $n=4785$ )
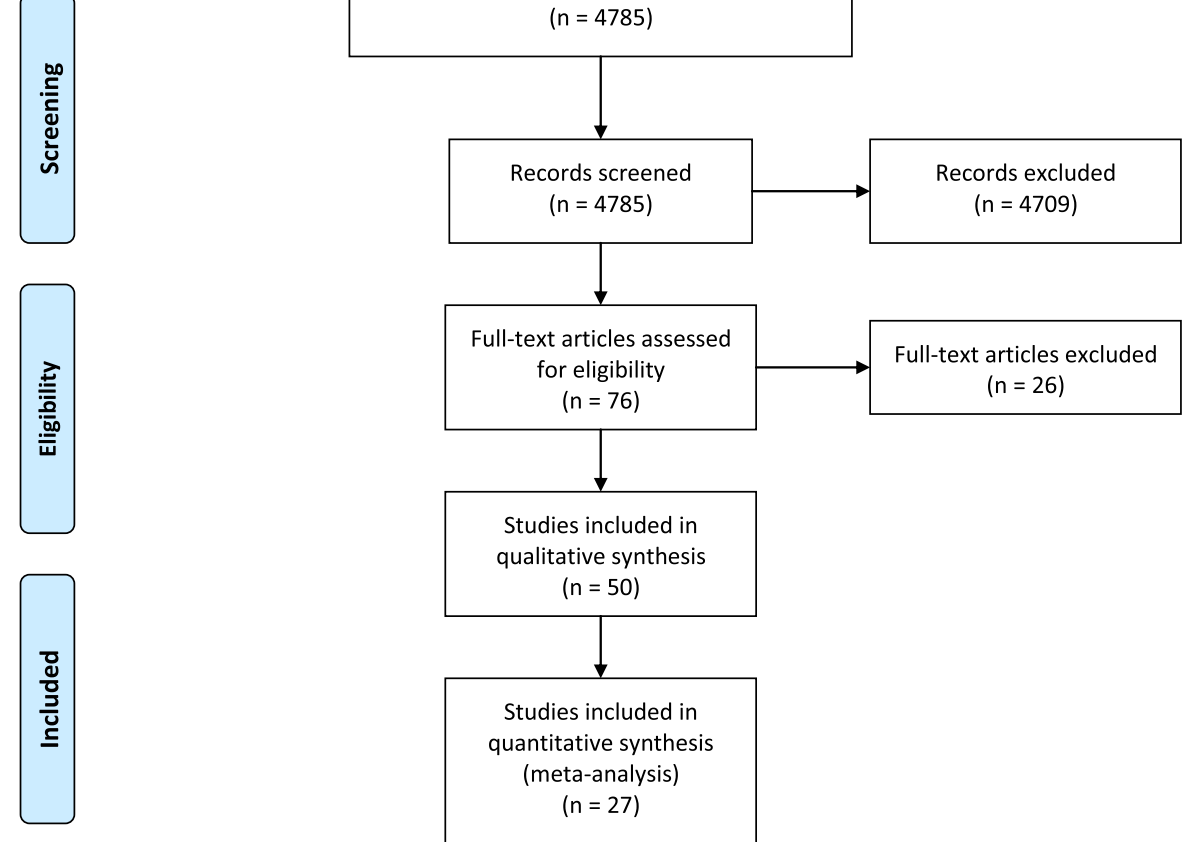

Fig. 1 PRISMA flow diagram 
Table 1 characteristics and summary of findings in the included studies

\begin{tabular}{|c|c|c|c|c|c|c|c|c|c|}
\hline \multirow[t]{2}{*}{ Study } & \multirow[t]{2}{*}{$\begin{array}{l}\text { Study } \\
\text { design }\end{array}$} & \multirow[t]{2}{*}{ Setting } & \multirow[t]{2}{*}{$\begin{array}{l}\text { Study } \\
\text { method }\end{array}$} & \multirow[t]{2}{*}{ Sample size } & \multirow[t]{2}{*}{$\begin{array}{l}\text { Mean } \\
\text { age } \pm S D\end{array}$} & \multirow[t]{2}{*}{$\begin{array}{l}\text { Female: } \\
\text { Male }\end{array}$} & \multirow[t]{2}{*}{$\begin{array}{l}\text { Thyroid } \\
\text { cancer type }\end{array}$} & \multicolumn{2}{|c|}{$\begin{array}{l}\text { Rate of Hashimoto's } \\
\text { thyroiditis (in percent) }\end{array}$} \\
\hline & & & & & & & & $\begin{array}{l}\text { Thyroid } \\
\text { cancer }\end{array}$ & $\begin{array}{l}\text { Control } \\
\text { group }\end{array}$ \\
\hline $\begin{array}{l}\text { Alcântara- } \\
\text { Jones } 2015 \\
{[12]}\end{array}$ & $\begin{array}{l}\text { Retrospec- } \\
\text { tive }\end{array}$ & Brazil & $\begin{array}{l}\text { Thyroidec- } \\
\text { tomy }\end{array}$ & 49 & 48.5 & $30: 3$ & Papillary & $\begin{array}{l}27.27 \\
(9 / 33)\end{array}$ & $\begin{array}{l}31.25 \\
(5 / 16)\end{array}$ \\
\hline $\begin{array}{l}\text { Zeng } 2016 \\
{[13]}\end{array}$ & $\begin{array}{l}\text { Retrospec- } \\
\text { tive }\end{array}$ & China & $\begin{array}{l}\text { Thyroidec- } \\
\text { tomy }\end{array}$ & 619 & 45.9 & 484:135 & Papillary & $\begin{array}{l}35.86 \\
(222 / 619)\end{array}$ & \\
\hline $\begin{array}{l}\text { Campos } \\
2012[1]\end{array}$ & $\begin{array}{l}\text { Retrospec- } \\
\text { tive }\end{array}$ & Brazil & $\begin{array}{l}\text { Thyroidec- } \\
\text { tomy }\end{array}$ & 315 & 44.9 & $34: 7$ & Papillary & $26.83(11 / 41)$ & $\begin{array}{l}1.12 \\
(3 / 268)\end{array}$ \\
\hline Ye 2013 [14] & $\begin{array}{l}\text { Retrospec- } \\
\text { tive }\end{array}$ & China & $\begin{array}{l}\text { Thyroidec- } \\
\text { tomy }\end{array}$ & 2052 & - & $828: 176$ & Papillary & $\begin{array}{l}18.63 \\
(187 / 1004)\end{array}$ & $6.42(66 / 1028)$ \\
\hline $\begin{array}{l}\text { Cipolla } 2005 \\
{[15]}\end{array}$ & $\begin{array}{l}\text { Retrospec- } \\
\text { tive }\end{array}$ & Italy & $\begin{array}{l}\text { Thyroidec- } \\
\text { tomy }\end{array}$ & 178 & - & $68: 21$ & Papillary & $\begin{array}{l}26.76 \\
(19 / 71)\end{array}$ & \\
\hline \multirow[t]{2}{*}{$\begin{array}{l}\text { Kim } 2011 \\
{[16]}\end{array}$} & $\begin{array}{l}\text { Retrospec- } \\
\text { tive }\end{array}$ & Korea & $\begin{array}{l}\text { Thyroidec- } \\
\text { tomy }\end{array}$ & 1329 & 47.5 & $821: 207$ & Papillary & $\begin{array}{l}29.86 \\
(307 / 1028)\end{array}$ & $9.64(24 / 249)$ \\
\hline & & & & & & $32: 20$ & Follicular & $\begin{array}{l}9.62 \\
(5 / 52)\end{array}$ & \\
\hline $\begin{array}{l}\text { Ahn } 2011 \\
{[17]}\end{array}$ & $\begin{array}{l}\text { Retrospec- } \\
\text { tive }\end{array}$ & Korea & $\begin{array}{l}\text { Thyroidec- } \\
\text { tomy }\end{array}$ & 303 & 42.8 & $225: 44$ & Papillary & $\begin{array}{l}21.56 \\
(58 / 269)\end{array}$ & \\
\hline \multirow[t]{2}{*}{$\begin{array}{l}\text { Huang } 2011 \\
\text { [18] }\end{array}$} & $\begin{array}{l}\text { Retrospec- } \\
\text { tive }\end{array}$ & China & $\begin{array}{l}\text { Thyroidec- } \\
\text { tomy }\end{array}$ & 1997 & 39.9 & $1450: 338$ & Papillary & $\begin{array}{l}4.75 \\
(85 / 1788)\end{array}$ & \\
\hline & & & & & & $153: 56$ & Follicular & $\begin{array}{l}3.83 \\
(8 / 209)\end{array}$ & \\
\hline $\begin{array}{l}\text { Lun } 2013 \\
{[19]}\end{array}$ & $\begin{array}{l}\text { Retrospec- } \\
\text { tive }\end{array}$ & China & $\begin{array}{l}\text { Thyroidec- } \\
\text { tomy }\end{array}$ & 2478 & 41.3 & 538:138 & Papillary & $\begin{array}{l}18.79 \\
(127 / 676)\end{array}$ & $\begin{array}{l}7.16 \\
(129 / 1802)\end{array}$ \\
\hline $\begin{array}{l}\text { Moshynska } \\
2008[20]\end{array}$ & $\begin{array}{l}\text { Retrospec- } \\
\text { tive }\end{array}$ & Canada & $\begin{array}{l}\text { Thyroidec- } \\
\text { tomy }\end{array}$ & 20 & - & - & Lymphoma & $\begin{array}{l}60 \\
(12 / 20)\end{array}$ & \\
\hline \multirow[t]{3}{*}{$\begin{array}{l}\text { Singh } 1999 \\
{[21]}\end{array}$} & $\begin{array}{l}\text { Retrospec- } \\
\text { tive }\end{array}$ & $\begin{array}{l}\text { United } \\
\text { States }\end{array}$ & $\begin{array}{l}\text { Thyroidec- } \\
\text { tomy }\end{array}$ & 453 & 41 & $267: 121$ & Papillary & $\begin{array}{l}14.69 \\
(57 / 388)\end{array}$ & \\
\hline & & & & & & - & Follicular & $\begin{array}{l}2.08 \\
(1 / 48)\end{array}$ & \\
\hline & & & & & & - & Lymphoma & $\begin{array}{l}5.88 \\
(1 / 17)\end{array}$ & \\
\hline $\begin{array}{l}\text { Zhang } 2014 \\
{[22]}\end{array}$ & $\begin{array}{l}\text { Retrospec- } \\
\text { tive }\end{array}$ & China & $\begin{array}{l}\text { Thyroidec- } \\
\text { tomy }\end{array}$ & 8524 & 43.1 & - & Papillary & $\begin{array}{l}28.46 \\
(592 / 2080)\end{array}$ & \\
\hline $\begin{array}{l}\text { Nemetz } 2011 \\
{[23]}\end{array}$ & $\begin{array}{l}\text { Retrospec- } \\
\text { tive }\end{array}$ & Brazil & $\begin{array}{l}\text { Thyroidec- } \\
\text { tomy }\end{array}$ & 52 & 51.3 & $48: 4$ & Papillary & $\begin{array}{l}32.69 \\
(17 / 52)\end{array}$ & \\
\hline $\begin{array}{l}\text { Jeong } 2012 \\
{[24]}\end{array}$ & $\begin{array}{l}\text { Retrospec- } \\
\text { tive }\end{array}$ & Korea & $\begin{array}{l}\text { Thyroidec- } \\
\text { tomy }\end{array}$ & 1357 & 44.5 & 1176:181 & Papillary & $\begin{array}{l}26.46 \\
(359 / 1357)\end{array}$ & \\
\hline $\begin{array}{l}\text { Kashima } \\
1998[25]\end{array}$ & $\begin{array}{l}\text { Retrospec- } \\
\text { tive }\end{array}$ & Japan & $\begin{array}{l}\text { Thyroidec- } \\
\text { tomy }\end{array}$ & 1533 & 42.6 & $1402: 131$ & Papillary & $\begin{array}{l}18.33 \\
(281 / 1533)\end{array}$ & \\
\hline $\begin{array}{l}\text { Kebebew } \\
2001[26]\end{array}$ & $\begin{array}{l}\text { Retrospec- } \\
\text { tive }\end{array}$ & $\begin{array}{l}\text { United } \\
\text { States }\end{array}$ & $\begin{array}{l}\text { Thyroidec- } \\
\text { tomy }\end{array}$ & 136 & 45.5 & $95: 41$ & Papillary & $\begin{array}{l}30.15 \\
(41 / 136)\end{array}$ & \\
\hline $\begin{array}{l}\text { Yoon } 2012 \\
{[27]}\end{array}$ & $\begin{array}{l}\text { Retrospec- } \\
\text { tive }\end{array}$ & Korea & $\begin{array}{l}\text { Thyroidec- } \\
\text { tomy }\end{array}$ & 195 & 45.9 & $166: 29$ & Papillary & $\begin{array}{l}28.72 \\
(56 / 195)\end{array}$ & \\
\hline $\begin{array}{l}\text { Graceffa } \\
2019[28]\end{array}$ & $\begin{array}{l}\text { Retrospec- } \\
\text { tive }\end{array}$ & Italy & $\begin{array}{l}\text { Thyroidec- } \\
\text { tomy }\end{array}$ & 305 & 50.6 & $258: 47$ & Papillary & $\begin{array}{l}28.6 \\
(36 / 126)\end{array}$ & $\begin{array}{l}7.7 \\
(11 / 142)\end{array}$ \\
\hline $\begin{array}{l}\text { Selek } 2016 \\
{[29]}\end{array}$ & $\begin{array}{l}\text { Retrospec- } \\
\text { tive }\end{array}$ & Turkey & $\begin{array}{l}\text { Thyroidec- } \\
\text { tomy }\end{array}$ & 870 & $47 \pm 12$ & & Papillary & $\begin{array}{l}30 \\
(172 / 577)\end{array}$ & $\begin{array}{l}31 \\
(90 / 293)\end{array}$ \\
\hline $\begin{array}{l}\text { Topaloglu } \\
2016 \text { [30] }\end{array}$ & $\begin{array}{l}\text { Retrospec- } \\
\text { tive }\end{array}$ & Turkey & $\begin{array}{l}\text { Thyroidec- } \\
\text { tomy }\end{array}$ & 427 & $\begin{array}{l}\text { Malignant: } \\
49.10 \pm 12.23 \\
\text { Benign: } \\
47.78 \pm 12.39\end{array}$ & $341: 86$ & Papillary & $\begin{array}{l}38.4 \\
(73 / 190)\end{array}$ & $\begin{array}{l}29.5 \\
(70 / 237)\end{array}$ \\
\hline $\begin{array}{l}\text { Zeng } 2018 \\
\text { [31] }\end{array}$ & $\begin{array}{l}\text { Retrospec- } \\
\text { tive }\end{array}$ & China & $\begin{array}{l}\text { Thyroidec- } \\
\text { tomy }\end{array}$ & 258 & $17.31 \pm 3.21$ & $212: 46$ & Papillary & $\begin{array}{l}17.8 \\
(23 / 129)\end{array}$ & $\begin{array}{l}1.6 \\
(2 / 129)\end{array}$ \\
\hline
\end{tabular}


Table 1 (continued)

\begin{tabular}{|c|c|c|c|c|c|c|c|c|c|}
\hline \multirow[t]{2}{*}{ Study } & \multirow[t]{2}{*}{$\begin{array}{l}\text { Study } \\
\text { design }\end{array}$} & \multirow[t]{2}{*}{ Setting } & \multirow[t]{2}{*}{$\begin{array}{l}\text { Study } \\
\text { method }\end{array}$} & \multirow[t]{2}{*}{ Sample size } & \multirow[t]{2}{*}{$\begin{array}{l}\text { Mean } \\
\text { age } \pm S D\end{array}$} & \multirow[t]{2}{*}{$\begin{array}{l}\text { Female: } \\
\text { Male }\end{array}$} & \multirow[t]{2}{*}{$\begin{array}{l}\text { Thyroid } \\
\text { cancer type }\end{array}$} & \multicolumn{2}{|c|}{$\begin{array}{l}\text { Rate of Hashimoto's } \\
\text { thyroiditis (in percent) }\end{array}$} \\
\hline & & & & & & & & $\begin{array}{l}\text { Thyroid } \\
\text { cancer }\end{array}$ & $\begin{array}{l}\text { Control } \\
\text { group }\end{array}$ \\
\hline $\begin{array}{l}\text { Osorio } 2019 \\
{[7]}\end{array}$ & $\begin{array}{l}\text { Retrospec- } \\
\text { tive }\end{array}$ & Colombia & $\begin{array}{l}\text { Thyroidec- } \\
\text { tomy }\end{array}$ & 1136 & $47.5 \pm 14.3$ & 1047: 89 & Papillary & $\begin{array}{l}24 \\
(44 / 183)\end{array}$ & $\begin{array}{l}13.11 \\
(125 / 953)\end{array}$ \\
\hline $\begin{array}{l}\text { Youssef } \\
\text { Mohamed } \\
2020 \text { [32] }\end{array}$ & $\begin{array}{l}\text { Retrospec- } \\
\text { tive }\end{array}$ & Egypt & $\begin{array}{l}\text { Thyroidec- } \\
\text { tomy }\end{array}$ & 80 & - & $22: 58$ & Papillary & $\begin{array}{l}20 \\
16 / 80\end{array}$ & \\
\hline \multirow[t]{4}{*}{$\begin{array}{l}\text { JNawarathna } \\
2018 \text { [33] }\end{array}$} & $\begin{array}{l}\text { Retrospec- } \\
\text { tive }\end{array}$ & Sri Lanka & $\begin{array}{l}\text { Thyroidec- } \\
\text { tomy }\end{array}$ & 684 & $48 \pm 12.5$ & $611: 73$ & Papillary & \multicolumn{2}{|c|}{ OR: 0.867 (0.25-2.99) } \\
\hline & & & & & & & \multirow[t]{3}{*}{ Follicular } & \multicolumn{2}{|c|}{ OR: $1.02(0.22-4.58)$} \\
\hline & & & & & & & & \multicolumn{2}{|c|}{$\begin{array}{l}\text { Rate of thyroid cancer (in } \\
\text { percent) }\end{array}$} \\
\hline & & & & & & & & $\begin{array}{l}\text { Hashimoto's } \\
\text { thyroiditis }\end{array}$ & $\begin{array}{l}\text { Control } \\
\text { group }\end{array}$ \\
\hline $\begin{array}{l}\text { Repplinger } \\
2008[34]\end{array}$ & $\begin{array}{l}\text { Retrospec- } \\
\text { tive }\end{array}$ & $\begin{array}{l}\text { United } \\
\text { States }\end{array}$ & $\begin{array}{l}\text { Thyroidec- } \\
\text { tomy }\end{array}$ & 1198 & & $215: 77$ & Papillary & $\begin{array}{l}29.03 \\
(63 / 217)\end{array}$ & $\begin{array}{l}23.34 \\
(229 / 981)\end{array}$ \\
\hline \multirow[t]{2}{*}{$\begin{array}{l}\text { Paparodis } \\
2014 \text { [35] }\end{array}$} & $\begin{array}{l}\text { Retrospec- } \\
\text { tive }\end{array}$ & $\begin{array}{l}\text { United } \\
\text { States }\end{array}$ & $\begin{array}{l}\text { Thyroidec- } \\
\text { tomy }\end{array}$ & 2718 & & & Papillary & $\begin{array}{l}42.68 \\
(242 / 567)\end{array}$ & $\begin{array}{l}26.27 \\
(565 / 2151)\end{array}$ \\
\hline & & & & & & & Follicular & $\begin{array}{l}1.76 \\
(10 / 567)\end{array}$ & $\begin{array}{l}2.14 \\
(46 / 2151)\end{array}$ \\
\hline $\begin{array}{l}\text { Anil } 2010 \\
{[36]}\end{array}$ & Prospective & Turkey & FNA & 715 & & & Papillary & $\begin{array}{l}1.22 \\
(2 / 164)\end{array}$ & $\begin{array}{l}3.45 \\
(19 / 551)\end{array}$ \\
\hline $\begin{array}{l}\text { Konturek } \\
2013[37]\end{array}$ & $\begin{array}{l}\text { Retrospec- } \\
\text { tive }\end{array}$ & Poland & $\begin{array}{l}\text { Thyroidec- } \\
\text { tomy }\end{array}$ & 7545 & 53.5 & & Papillary & $\begin{array}{l}23.45 \\
(106 / 452)\end{array}$ & $\begin{array}{l}7.47 \\
(530 / 7093)\end{array}$ \\
\hline \multirow[t]{2}{*}{$\begin{array}{l}\text { Mukasa } 2011 \\
\text { [38] }\end{array}$} & $\begin{array}{l}\text { Retrospec- } \\
\text { tive }\end{array}$ & Japan & FNA & 2036 & & & Papillary & $\begin{array}{l}1.77 \\
(36 / 2036)\end{array}$ & \\
\hline & & & & & & & Lymphoma & $\begin{array}{l}0.10 \\
(2 / 2036)\end{array}$ & \\
\hline $\begin{array}{l}\text { Matesa-Anic } \\
2009[39]\end{array}$ & $\begin{array}{l}\text { Retrospec- } \\
\text { tive }\end{array}$ & Croatia & FNA & 10,508 & 50 & $236: 33$ & Papillary & $\begin{array}{l}1.95 \\
(42 / 2156)\end{array}$ & $\begin{array}{l}2.72 \\
(227 / 8352)\end{array}$ \\
\hline $\begin{array}{l}\text { Dailey } 1955 \\
\text { [5] }\end{array}$ & Prospective & $\begin{array}{l}\text { United } \\
\text { States }\end{array}$ & $\begin{array}{l}\text { Thyroidec- } \\
\text { tomy }\end{array}$ & 2336 & 37.5 & & Papillary & $\begin{array}{l}10.43 \\
(29 / 278)\end{array}$ & \\
\hline \multirow[t]{3}{*}{$\begin{array}{l}\text { Larson } 2007 \\
\text { [40] }\end{array}$} & $\begin{array}{l}\text { Retrospec- } \\
\text { tive }\end{array}$ & $\begin{array}{l}\text { United } \\
\text { States }\end{array}$ & $\begin{array}{l}\text { Thyroidec- } \\
\text { tomy }\end{array}$ & 812 & 41 & $142: 37$ & Papillary & $\begin{array}{l}34.7 \\
(34 / 98)\end{array}$ & $\begin{array}{l}20.4 \\
(145 / 710)\end{array}$ \\
\hline & & & & & & $16: 5$ & Follicular & $\begin{array}{l}9.2 \\
(9 / 98)\end{array}$ & $\begin{array}{l}1.69 \\
(12 / 710)\end{array}$ \\
\hline & & & & & & $3: 0$ & Anaplastic & $\begin{array}{l}1.02 \\
(1 / 98)\end{array}$ & $\begin{array}{l}0.28 \\
(2 / 710)\end{array}$ \\
\hline \multirow[t]{3}{*}{$\begin{array}{l}\text { Zayed } 2015 \\
\text { [41] }\end{array}$} & $\begin{array}{l}\text { Retrospec- } \\
\text { tive }\end{array}$ & Jordan & $\begin{array}{l}\text { Thyroidec- } \\
\text { tomy }\end{array}$ & 180 & 51.3 & $9: 6$ & Medullary & $\begin{array}{l}3.85 \\
(3 / 78)\end{array}$ & $\begin{array}{l}1.53 \\
(12 / 785)\end{array}$ \\
\hline & & & & & & $102: 35$ & Papillary & $\begin{array}{l}10.26 \\
(8 / 78)\end{array}$ & $\begin{array}{l}16.43 \\
(129 / 785)\end{array}$ \\
\hline & & & & & & $18: 9$ & Follicular & $\begin{array}{l}0 \\
(0 / 78)\end{array}$ & $\begin{array}{l}3.44 \\
(27 / 785)\end{array}$ \\
\hline \multirow[t]{4}{*}{ Gul 2010 [42] } & $\begin{array}{l}\text { Retrospec- } \\
\text { tive }\end{array}$ & Turkey & $\begin{array}{l}\text { Thyroidec- } \\
\text { tomy }\end{array}$ & 613 & 43 & & Papillary & $43.48(40 / 92)$ & $\begin{array}{l}25.14 \\
(131 / 521)\end{array}$ \\
\hline & & & & & & & Follicular & $\begin{array}{l}1.09 \\
(1 / 92)\end{array}$ & $\begin{array}{l}1.92 \\
(10 / 521)\end{array}$ \\
\hline & & & & & & & Medullary & $\begin{array}{l}0 \\
(0 / 92)\end{array}$ & $\begin{array}{l}0.96 \\
(5 / 521)\end{array}$ \\
\hline & & & & & & & Lymphoma & $\begin{array}{l}1.09 \\
(1 / 92)\end{array}$ & $\begin{array}{l}0 \\
(0 / 521)\end{array}$ \\
\hline $\begin{array}{l}\text { Mazokopakis } \\
2010 \text { [43] }\end{array}$ & $\begin{array}{l}\text { Retrospec- } \\
\text { tive }\end{array}$ & Greece & $\begin{array}{l}\text { Thyroidec- } \\
\text { tomy }\end{array}$ & 140 & 49.3 & $25: 7$ & Papillary & $\begin{array}{l}28.57 \\
(12 / 42)\end{array}$ & $\begin{array}{l}20.41 \\
(20 / 98)\end{array}$ \\
\hline
\end{tabular}


Table 1 (continued)

\begin{tabular}{|c|c|c|c|c|c|c|c|c|c|}
\hline \multirow[t]{2}{*}{ Study } & \multirow[t]{2}{*}{$\begin{array}{l}\text { Study } \\
\text { design }\end{array}$} & \multirow[t]{2}{*}{ Setting } & \multirow[t]{2}{*}{$\begin{array}{l}\text { Study } \\
\text { method }\end{array}$} & \multirow[t]{2}{*}{ Sample size } & \multirow[t]{2}{*}{$\begin{array}{l}\text { Mean } \\
\text { age } \pm \text { SD }\end{array}$} & \multirow[t]{2}{*}{$\begin{array}{l}\text { Female: } \\
\text { Male }\end{array}$} & \multirow[t]{2}{*}{$\begin{array}{l}\text { Thyroid } \\
\text { cancer type }\end{array}$} & \multicolumn{2}{|c|}{$\begin{array}{l}\text { Rate of Hashimoto's } \\
\text { thyroiditis (in percent) }\end{array}$} \\
\hline & & & & & & & & $\begin{array}{l}\text { Thyroid } \\
\text { cancer }\end{array}$ & $\begin{array}{l}\text { Control } \\
\text { group }\end{array}$ \\
\hline $\begin{array}{l}\text { Sclafani } 1993 \\
\text { [44] }\end{array}$ & $\begin{array}{l}\text { Retrospec- } \\
\text { tive }\end{array}$ & $\begin{array}{l}\text { United } \\
\text { States }\end{array}$ & $\begin{array}{l}\text { Thyroidec- } \\
\text { tomy }\end{array}$ & 48 & 51.7 & & Papillary & $\begin{array}{l}12.5 \\
(6 / 48)\end{array}$ & \\
\hline $\begin{array}{l}\text { Peterson } \\
1957[45]\end{array}$ & $\begin{array}{l}\text { Retrospec- } \\
\text { tive }\end{array}$ & $\begin{array}{l}\text { United } \\
\text { States }\end{array}$ & $\begin{array}{l}\text { Thyroidec- } \\
\text { tomy }\end{array}$ & 757 & & & Papillary & $\begin{array}{l}2.60 \\
(2 / 77)\end{array}$ & $\begin{array}{l}2.06 \\
(14 / 680)\end{array}$ \\
\hline \multirow[t]{5}{*}{$\begin{array}{l}\text { Zhang } 2014 \\
\text { [46] }\end{array}$} & $\begin{array}{l}\text { Retrospec- } \\
\text { tive }\end{array}$ & China & $\begin{array}{l}\text { Thyroidec- } \\
\text { tomy }\end{array}$ & 647 & 43.3 & & Papillary & $\begin{array}{l}37.96 \\
(41 / 108)\end{array}$ & $\begin{array}{l}17.25 \\
(93 / 539)\end{array}$ \\
\hline & & & & & & & Follicular & $\begin{array}{l}0 \\
(0 / 108)\end{array}$ & $\begin{array}{l}0.37 \\
(2 / 539)\end{array}$ \\
\hline & & & & & & & Medullary & $\begin{array}{l}1.85 \\
(2 / 108)\end{array}$ & $\begin{array}{l}0.37 \\
(2 / 539)\end{array}$ \\
\hline & & & & & & & Anaplastic & $\begin{array}{l}0 \\
(0 / 108)\end{array}$ & $\begin{array}{l}0.56 \\
(3 / 539)\end{array}$ \\
\hline & & & & & & & Lymphoma & $\begin{array}{l}0.93 \\
(1 / 108)\end{array}$ & $\begin{array}{l}0 \\
(0 / 539)\end{array}$ \\
\hline \multirow[t]{3}{*}{$\begin{array}{l}\text { Holm } 1985 \\
{[47]}\end{array}$} & Prospective & Sweden & FNA & 1656 & & & Papillary & $\begin{array}{l}0.12 \\
(1 / 829)\end{array}$ & $\begin{array}{l}0.12 \\
(1 / 829)\end{array}$ \\
\hline & & & & & & & Follicular & $\begin{array}{l}0.12 \\
(1 / 829)\end{array}$ & $\begin{array}{l}0.12 \\
(1 / 829)\end{array}$ \\
\hline & & & & & & & Lymphoma & $\begin{array}{l}0.48 \\
(4 / 829)\end{array}$ & $\begin{array}{l}0 \\
(0 / 829)\end{array}$ \\
\hline \multirow[t]{2}{*}{$\begin{array}{l}\text { Moris } 2019 \\
{[48]}\end{array}$} & prospective & $\begin{array}{l}\text { United } \\
\text { States }\end{array}$ & $\begin{array}{l}\text { Thyroidec- } \\
\text { tomy }\end{array}$ & 9851 & $52.2 \pm 15$ & $\begin{array}{l}\text { 8263: } \\
1588\end{array}$ & Undefined & $\begin{array}{l}22.8 \\
(606 / 2651)\end{array}$ & $\begin{array}{l}15.4 \\
(1105 / 7200)\end{array}$ \\
\hline & & & FNA & & & & Undefined & $\begin{array}{l}7.3 \\
(284 / 3895)\end{array}$ & $\begin{array}{l}4.7 \\
(473 / 10168)\end{array}$ \\
\hline \multirow[t]{2}{*}{$\begin{array}{l}\text { Jackson } 2020 \\
{[49]}\end{array}$} & retrospective & $\begin{array}{l}\text { United } \\
\text { States }\end{array}$ & $\begin{array}{l}\text { Thyroidec- } \\
\text { tomy }\end{array}$ & 359 & & & $\begin{array}{l}\text { Incidental } \\
\text { thyroid } \\
\text { cancer }\end{array}$ & $\begin{array}{l}15 \\
(8 / 52)\end{array}$ & $\begin{array}{l}10 \\
(31 / 307)\end{array}$ \\
\hline & & & & & & & $\begin{array}{l}\text { Thyroid } \\
\text { cancer }\end{array}$ & $37(19 / 52)$ & $37(114 / 307)$ \\
\hline $\begin{array}{l}\text { Keskin } 2016 \\
{[50]}\end{array}$ & prospective & Turkey & $\begin{array}{l}\text { FNA and } \\
\text { Thyroidec- } \\
\text { tomy }\end{array}$ & 300 & $12.1 \pm 3.1$ & 238: 62 & Papillary & $\begin{array}{l}0.66 \\
(2 / 300)\end{array}$ & \\
\hline Liu2017 [51] & retrospective & China & $\begin{array}{l}\text { Thyroidec- } \\
\text { tomy }\end{array}$ & 927 & $46 \pm 0$ & $\begin{array}{l}706: \\
221\end{array}$ & Papillary & & \\
\hline $\begin{array}{l}\text { Radetti } 2019 \\
\text { [52] }\end{array}$ & prospective & Italy & FNA & 904 & $10.6 \pm 3.2$ & $\begin{array}{l}709: \\
195\end{array}$ & Papillary & $\begin{array}{l}1.1 \\
(10 / 904)\end{array}$ & \\
\hline $\begin{array}{l}\text { Won2018 } \\
\text { [53] }\end{array}$ & retrospective & Korea & FNA & 89 & $\begin{array}{l}11.1 \pm \\
3.7\end{array}$ & $76: 13$ & Papillary & $\begin{array}{l}7.9 \\
(7 / 89)\end{array}$ & \\
\hline \multirow[t]{4}{*}{ Boi 2017 [54] } & retrospective & Italy & FNA & 645 & & & & $\begin{array}{l}28.9 \\
(44 / 152)\end{array}$ & $\begin{array}{l}7.4 \\
(12 / 161)\end{array}$ \\
\hline & & & $\begin{array}{l}\text { Thyroidec- } \\
\text { tomy }\end{array}$ & & & & Papillary & $\begin{array}{l}64.3 \\
(45 / 70)\end{array}$ & $\begin{array}{l}35.1 \\
(13 / 37)\end{array}$ \\
\hline & & & $\begin{array}{l}\text { Thyroidec- } \\
\text { tomy }\end{array}$ & & & & Follicular & $\begin{array}{l}4.3 \\
(3 / 70)\end{array}$ & $\begin{array}{l}5.4 \\
(2 / 37)\end{array}$ \\
\hline & & & $\begin{array}{l}\text { Thyroidec- } \\
\text { tomy }\end{array}$ & & & & Medullary & $2.8(2 / 70)$ & $\begin{array}{l}2.7 \\
(1 / 37)\end{array}$ \\
\hline \multirow[t]{2}{*}{$\begin{array}{l}\text { Gabalec } \\
2016[55]\end{array}$} & retrospective & $\begin{array}{l}\text { Czech } \\
\text { republic }\end{array}$ & $\begin{array}{l}\text { Thyroidec- } \\
\text { tomy }\end{array}$ & 4947 & & & Undefined & $\begin{array}{l}29.5 \\
(26 / 88)\end{array}$ & $\begin{array}{l}15.2 \\
(231 / 1515)\end{array}$ \\
\hline & & & FNA & & & & & $\begin{array}{l}14.2 \\
(85 / 592)\end{array}$ & $\begin{array}{l}15.2 \\
(662 / 4348)\end{array}$ \\
\hline \multirow[t]{2}{*}{$\begin{array}{l}\text { Büyükaşi } \\
2011 \text { [56] }\end{array}$} & retrospective & Turkey & $\begin{array}{l}\text { Thyroidec- } \\
\text { tomy }\end{array}$ & 917 & adult & $743: 174$ & $\begin{array}{l}\text { All Cancer } \\
\text { Types }\end{array}$ & $\begin{array}{l}19.4 \\
(15 / 77)\end{array}$ & $\begin{array}{l}9.8 \\
(82 / 840)\end{array}$ \\
\hline & & & & & & & Papillary & $\begin{array}{l}60.0 \\
(9 / 15)\end{array}$ & $\begin{array}{l}63.41 \\
(52 / 82)\end{array}$ \\
\hline
\end{tabular}


Table 1 (continued)

\begin{tabular}{|c|c|c|c|c|c|c|c|c|c|}
\hline \multirow[t]{2}{*}{ Study } & \multirow[t]{2}{*}{$\begin{array}{l}\text { Study } \\
\text { design }\end{array}$} & \multirow[t]{2}{*}{ Setting } & \multirow[t]{2}{*}{$\begin{array}{l}\text { Study } \\
\text { method }\end{array}$} & \multirow[t]{2}{*}{ Sample size } & \multirow[t]{2}{*}{$\begin{array}{l}\text { Mean } \\
\text { age } \pm S D\end{array}$} & \multirow[t]{2}{*}{$\begin{array}{l}\text { Female: } \\
\text { Male }\end{array}$} & \multirow[t]{2}{*}{$\begin{array}{l}\text { Thyroid } \\
\text { cancer type }\end{array}$} & \multicolumn{2}{|c|}{$\begin{array}{l}\text { Rate of Hashimoto's } \\
\text { thyroiditis (in percent) }\end{array}$} \\
\hline & & & & & & & & $\begin{array}{l}\text { Thyroid } \\
\text { cancer }\end{array}$ & $\begin{array}{l}\text { Control } \\
\text { group }\end{array}$ \\
\hline & & & & & & & Follicular & $\begin{array}{l}6.6 \\
(1 / 15)\end{array}$ & $\begin{array}{l}13.41 \\
(11 / 82)\end{array}$ \\
\hline & & & & & & & Medullary & $\begin{array}{l}20.0 \\
(3 / 15)\end{array}$ & $\begin{array}{l}4.87 \\
(4 / 82)\end{array}$ \\
\hline $\begin{array}{l}\text { Chen } 2013 \\
\text { [57] }\end{array}$ & prospective & Taiwan & & 7605 & adult & $6845: 755$ & & 1.58 & 0.14 \\
\hline $\begin{array}{l}\text { Cipolla } 2005 \\
\text { [15] }\end{array}$ & $\begin{array}{l}\text { Retrospec- } \\
\text { tive }\end{array}$ & Italy & $\begin{array}{l}\text { Thyroidec- } \\
\text { tomy }\end{array}$ & 178 & - & $68: 21$ & Papillary & $\begin{array}{l}27.6 \\
(13 / 47)\end{array}$ & \\
\hline $\begin{array}{l}\text { Zhang } 2014 \\
\text { [22] }\end{array}$ & $\begin{array}{l}\text { Retrospec- } \\
\text { tive }\end{array}$ & China & $\begin{array}{l}\text { Thyroidec- } \\
\text { tomy }\end{array}$ & 8524 & 43.1 & - & Papillary & $\begin{array}{l}14.24 \\
(247 / 839)\end{array}$ & $\begin{array}{l}70 \\
(592 / 839)\end{array}$ \\
\hline \multirow[t]{3}{*}{$\begin{array}{l}\text { Graceffa } \\
2019[28]\end{array}$} & $\begin{array}{l}\text { Retrospec- } \\
\text { tive }\end{array}$ & Italy & $\begin{array}{l}\text { Thyroidec- } \\
\text { tomy }\end{array}$ & 305 & 50.6 & $258: 47$ & Papillary & $\begin{array}{l}28.83 \\
(47 / 163)\end{array}$ & \\
\hline & & & & & & & Follicular & $\begin{array}{l}1.84 \\
(3 / 163)\end{array}$ & \\
\hline & & & & & & & Medullary & $\begin{array}{l}0.61 \\
(1 / 163)\end{array}$ & \\
\hline $\begin{array}{l}\text { Uhliarova } \\
2017[58]\end{array}$ & prospective & Slovakia & $\begin{array}{l}\text { Thyroidec- } \\
\text { tomy }\end{array}$ & 2117 & $11.1 \pm 3.7$ & 1738: 379 & $\begin{array}{l}\text { All cancer } \\
\text { types }\end{array}$ & $\begin{array}{l}83.64 \%) \\
(266 / 318)\end{array}$ & \\
\hline
\end{tabular}

ranged between 0 to $20 \%$. The meta-analysis of 5 studies with an appropriate control group, reached 2.70 OR for this outcome (95\% CI: 1.20 to 6.07 ; $\mathrm{I}^{2}$ test for heterogeneity: $0 \%)$ and the difference between the groups was significant $(p=0.01)$.

\section{Lymphoma}

6 studies investigated the relation between HT and lymphoma and the rate of HT in the lymphoma group was 5.88 and $60 \%$ in two studies. The range of lymphoma in HT was between 0.1 to $1.09 \%$. The meta-analysis of 3 studies concerning this outcome reached 12.93 OR (95\% CI: 2.15 to 77.63 ; $\mathrm{I}^{2}$ test for heterogeneity: $0 \%$ ) and the difference between these groups was significant $(p=0.01)$.

\section{Anaplastic thyroid cancer}

Only two studies assessed the relation between anaplastic thyroid cancer (ATC) and HT and the rate of TC in the HT group was 0 and $1.02 \%$ in these studies. The metaanalysis reached 1.92 OR (95\% CI: 1.90 to 0.29 ; $\mathrm{I}^{2}$ test for heterogeneity: $0 \%$ ) and the difference between groups was not statistically significant $(p=0.05)$.

\section{All cancer types}

Twenty seven studies had an appropriate control group which allowed us to calculate the OR and include them in the meta-analysis. The results showed 1.36 OR (95\% CI: 1.05 to 1.77 ; $\mathrm{I}^{2}$ test for heterogeneity: $93.66 \%$ ) and there was a significant difference between case and control groups in terms of incidence of TCs $(p=0.01)$.

\section{Risk of Bias}

The RoB assessment based on the JBI checklist is presented in Fig. 3. Based on our assessment, appropriately dealing with confounding factors was the most prevalent source of bias in included studies. The appropriate and complete follow-up period was the other source of bias in these studies. Generally, there is a concerning risk of bias in these studies which can affect these outcomes. The details of the RoB assessment are presented in Supplementary material 1 .

\section{Discussion}

This study investigated the possible relationship between HT and different types of TCs. The results indicated a significant correlation between HT and thyroid malignancies in particular with PTC, MTC, lymphoma but not with ATC and FTC. Also, this study found a significant association between HT and thyroid malignancies.

As the most common cause of hypothyroidism in developed countries [59], the role of HT in developing thyroid malignancies, should be considered by the clinicians. As fine needle aspiration (FNA) has poor accuracy in the diagnosis of TCs in patients with thyroiditis, diagnosis of TC in the presence of HT is challenging. Previous studies have found a better prognosis for TC in case of coexistence of HT, because of earlier diagnosis based 


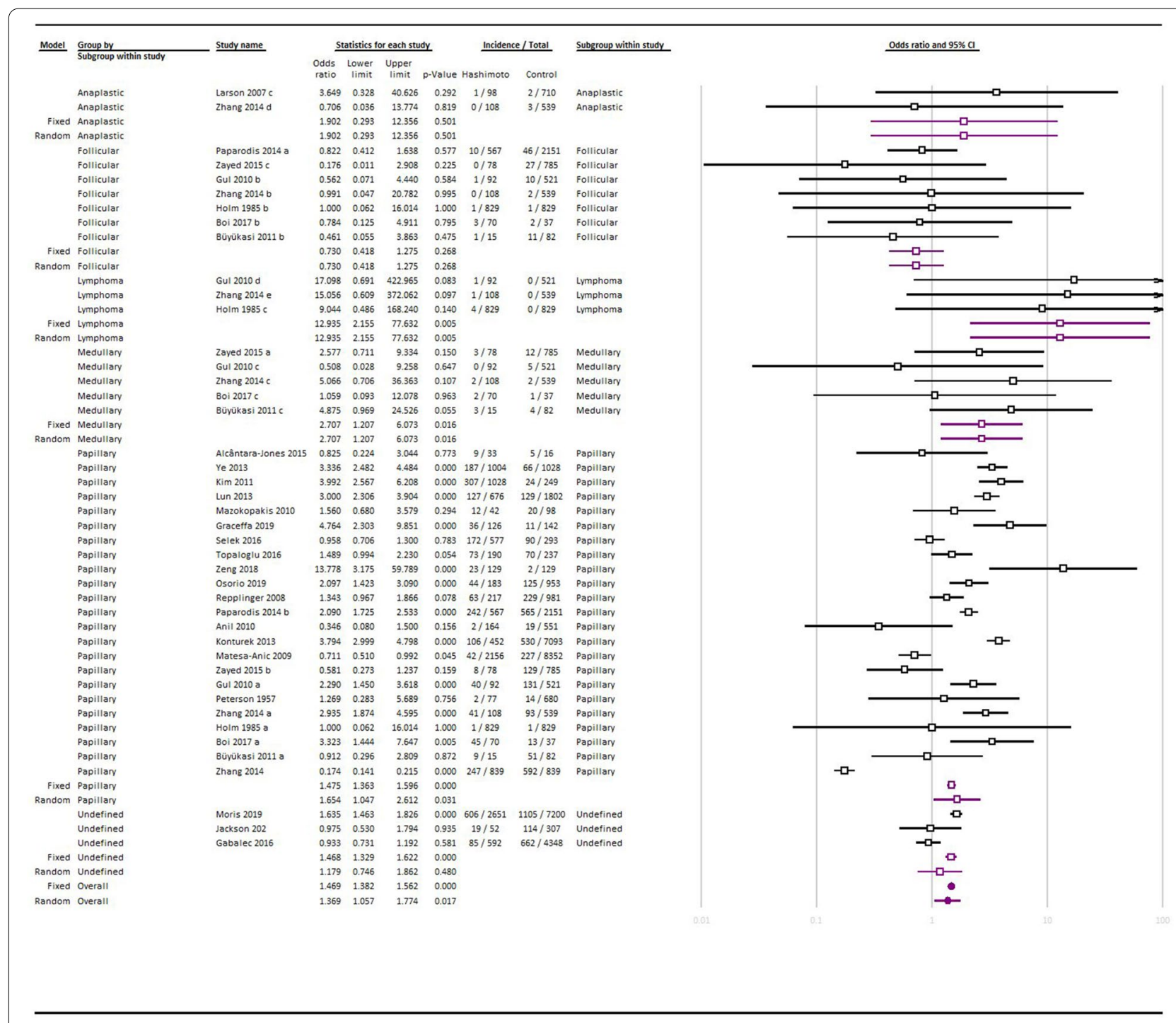

Fig. 2 forest plot of the studies included in the meta-analysis

on routine medical follow-up [18]. Moreover, a less aggressive form of malignancy in PTC patients in the top of HT has been reported, though but this conclusion was associated with controversies in an endemic area of iodine deficiency goiter [60].

Despite multiple hypotheses in this regard, the underlying mechanism of developing malignancies in HT patients is not fully understood [61, 62]. One of these mechanisms may rely on the inflammatory process in $\mathrm{HT}$. Inflammatory reactions create free radical oxygen, resulting in DNA damage and mutations that finally cause the development of PTC [9]. Another hypothesis states that malignant transformation is caused by increased levels of TSH that stimulate thyroid tissue epithelial proliferation [61]. A recently published study assessed the prognostic value of FOXP3 in PTC and the difference in its expression in concomitant HT. FOXP3 is a PTC-related marker and its expression by HT infiltrating lymphocytes suggested a relationship between HT and PTC [32].

Despite the historical discussion about the possible role of HT in developing TCs, current guidelines didn't accept HT as a risk factor for developing thyroid malignancies [63]. Some experts believe that a good prognosis of TCs and particularly PTC, as the most incident thyroid malignancy, leads to a decrease in allocation of resources toward designing and conducting well-designed studies to identify predictive factors and improving the management of outcomes [64]. The controversial outcomes of the studies highlighted a need for more prospective studies with appropriate control groups and considering 


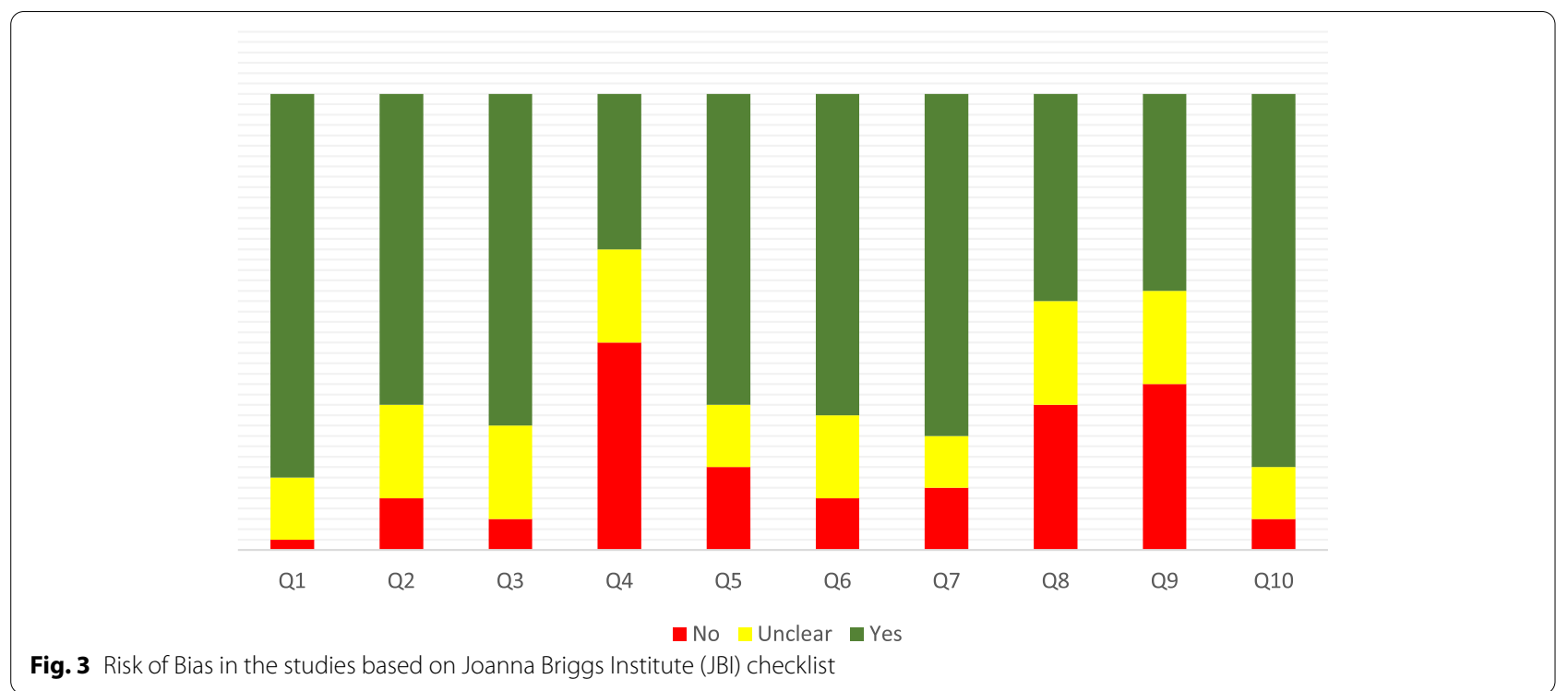

the possible cofounding factors to reach more reliable evidence.

Our meta-analysis as the most reliable evidence in this regard found a significant association between HT and MTC based on 5 published studies. This finding is obtained based on a retrospective point of view and only 11 cases of MTC were reported in 526 investigated cases of HT in our included studies. MTC is the third most common TC that originates from the parafollicular cells with an unfavorable prognosis $[65,66]$. Previously the reports of this relation were limited to case reports [6772]. One of the suggested pathophysiological bases for this relation is the occurrence of HT in response to MTC, so future prospective studies can give better insight in this regard. Also, the results of Zayed et al. only found such an association only in female patients [41], which should be more investigated in future studies.

One of the limitations of this study was the high level of heterogeneity between different studies. These differences can arise from multiple sources. Differences in pathological interpretation of HT, genetic factors, diagnostic methods for thyroid malignancies including the FNA and total thyroidectomy can cause variations in the reported rate of coexistence of HT and TC in our included studies. Besides, the variation in OR can arise from differences in defining the control groups.

A comprehensive search in three major databases and the adding of hand searching results was one of the strengths of this study that led to full coverage of published studies that met our inclusion. Besides, carefully selecting and extracting the data, was the other strength of this systematic review. Unlike previous studies, we conducted our meta-analysis based on OR, therefore, the studies without an appropriate control group were excluded from our meta-analysis. This made the findings of our study more practical and obvious.

\section{Conclusion}

Based on the current knowledge, HT is associated with developing thyroid malignancies, particularly PTC, MTC, lymphoma but not with ATC and FTC. Studies with high RoB, the high level of heterogeneity between different studies, and the limited number of welldesigned prospective studies make the available evidence uncertain, so there is a need for more studies to reach more reliable conclusions.

\section{Abbreviations}

HT: Hashimoto thyroiditis; TC: Thyroid cancer; PTC: Papillary thyroid carcinoma; PRISMA: Preferred Reporting Items for Systematic Reviews and Meta-Analyses; RoB: Risk of bias; JBI: Joanna Briggs Institute; CMA: Comprehensive MetaAnalysis; OR: Odds ratio; FTC: Follicular thyroid cancer; MTC: Medullary thyroid cancer; ATC: Anaplastic thyroid cancer.

\section{Supplementary Information}

The online version contains supplementary material available at https://doi. org/10.1186/s13044-021-00117-x.

Additional file 1

\section{Acknowledgments}

The research protocol was approved and supported by Student Research Committee, Tabriz University of Medical Sciences (grant number: 64115).

\section{Conflict of interest}

None. 


\section{Authors' contributions}

PA and EN: Funding acquisition; Investigation; Resources; Project administration; Conceptualization AN: Formal analysis; Investigation; Methodology; Resources; Validation; Roles/Writing - original draft; VS*: Conceptualization; Data curation; Funding acquisition; Methodology; Writing - review \& editing. All authors read and approved the final manuscript.

\section{Funding}

This work was supported by Deputy for Research of Tabriz University of Medical Sciences.

\section{Availability of data and materials}

Not applicable.

\section{Declarations}

\section{Ethics approval and consent to participate}

The study protocol was approved by the ethics committee of Tabriz University of Medical Sciences (approval code: IR.TBZMED.VCR.REC.1399.434)

\section{Consent for publication}

Not applicable.

\section{Competing interests}

The authors declare no conflicts of interest.

\section{Author details}

'Student Research Committee, Tabriz University of Medical Sciences, Tabriz, Iran. ${ }^{2}$ Aging Research Institute, Tabriz University of Medical Sciences, Tabriz Iran. ${ }^{3}$ Research Center for Evidence-Based Medicine, Iranian EBM Centre: A Joanna Briggs Institute (JBI) Center of Excellence, Tabriz University of Medical Sciences, Tabriz, Iran. ${ }^{4}$ Endocrine Research Center, Tabriz University of Medical Sciences, Golgasht street, Tabriz, Iran.

Received: 20 May 2021 Accepted: 16 November 2021

\section{Published online: 03 December 2021}

\section{References}

1. Campos LA, Picado SM, Guimarães AV, Ribeiro DA, Dedivitis RA. Thyroid papillary carcinoma associated to Hashimoto's thyroiditis. Braz J Otorhinolaryngol. 2012;78(6):77-80.

2. Hashimoto H. Zur kenntniss der lymphomatosen veranderung der Schilddruse (Struma lymphomatosa). Arch Klin Chir. 1912;97:219-48.

3. Taylor PN, Albrecht D, Scholz A, Gutierrez-Buey G, Lazarus JH, Dayan CM, et al. Global epidemiology of hyperthyroidism and hypothyroidism. Nat Rev Endocrinol. 2018;14(5):301.

4. Balkwill F, Mantovani A. Inflammation and cancer: back to Virchow? Lancet. 2001;357(9255):539-45.

5. Me D, Lindsay S, Skahen R. Relation of thyroid neoplasms to hashimoto disease of the thyroid gland. AMA Arch Surg. 1955;70(2):291-7.

6. Resende de Paiva C, Grønhøj C, Feldt-Rasmussen U, CJFio v B. Association between Hashimoto's thyroiditis and thyroid cancer in 64,628 patients. Front Oncol. 2017:7:53.

7. Osorio C, Ibarra S, Arrieta J, Sarmiento M, Barrios D, Sierra L, et al. Association between chronic lymphocytic thyroiditis and papillary thyroid carcinoma: A retrospective study in surgical specimens. Rev Esp Patol. 2020;53(3):149-57.

8. Lai X, Xia Y, Zhang B, Li J, Jiang YJO. A meta-analysis of Hashimoto's thyroiditis and papillary thyroid carcinoma risk. Oncotarget. 2017:8(37):62414

9. Jankovic B, Le KT, Hershman JM, Metabolism. Hashimoto's thyroiditis and papillary thyroid carcinoma: is there a correlation? J Clin Endocrinol Metab. 2013;98(2):474-82

10. Moher D, Liberati A, Tetzlaff J, Altman DG. Preferred reporting items for systematic reviews and meta-analyses: the PRISMA statement. Int I Surg 2010:8(5):336-41.
11. Moola S, Munn Z, Tufanaru C, Aromataris E, Sears K, Sfetcu R, et al. Chapter 7: systematic reviews of etiology and risk, vol. 5: Joanna Briggs Institute Reviewer's Manual The Joanna Briggs Institute; 2017.

12. Alcântara-Jones DM, Alcântara-Nunes TF, Rocha BO, Oliveira RD, Santana ACP, Alcântara FT, et al. Is there any association between Hashimoto's thyroiditis and thyroid cancer? A retrospective data analysis. Radiol Bras. 2015:48(3):148-53.

13. Rc Z, Lp J, Ed C, Dong S, Yf C, Gl H, et al. Potential relationship between Hashimoto's thyroiditis and BRAFV600E mutation status in papillary thyroid cancer. Head Neck. 2016;38(S1):E1019-E25.

14. Ye Z-q, Gu D-n, Hu H-y, Zhou Y-I, Hu X-q, Zhang X-h. Hashimoto's thyroiditis, microcalcification and raised thyrotropin levels within normal range are associated with thyroid cancer. World J Surg Oncol. 2013;11(1):56.

15. Cipolla C, Sandonato L, Graceffa G, Fricano S, Torcivia A, Vieni S, et al. Hashimoto thyroiditis coexistent with papillary thyroid carcinoma. Am Surg. 2005;71(10):874-8.

16. Kim KW, Park YJ, Kim EH, Park SY, Park DJ, Ahn SH, et al. Elevated risk of papillary thyroid cancer in Korean patients with Hashimoto's thyroiditis. Head Neck. 2011;33(5):691-5.

17. Ahn D, Heo SJ, Park JH, Kim JH, Sohn JH, Park JY, et al. Clinical relationship between Hashimoto's thyroiditis and papillary thyroid cancer. Acta Oncol. 2011;50(8):1228-34.

18. Huang B-Y, Hseuh C, Chao T-C, Lin K-J, Lin J-D. Well-differentiated thyroid carcinoma with concomitant Hashimoto's thyroiditis present with less aggressive clinical stage and low recurrence. Endocr Pathol. 2011;22(3):144.

19. Lun Y, Wu X, Xia Q, Han Y, Zhang X, Liu Z, et al. Hashimoto's thyroiditis as a risk factor of papillary thyroid cancer may improve cancer prognosis. Otolaryngol Head Neck Surg. 2013;148(3):396-402.

20. Moshynska OV, Saxena A. Clonal relationship between Hashimoto thyroiditis and thyroid lymphoma. J Clin Pathol. 2008:61(4):438-44.

21. Singh B, Shaha AR, Trivedi H, Carew JF, Poluri A, Shah JP. Coexistent Hashimoto's thyroiditis with papillary thyroid carcinoma: impact on presentation, management, and outcome. Surgery. 1999;126(6):1070-7.

22. Zhang Y, Dai J, Wu T, Yang N, Yin Z. The study of the coexistence of Hashimoto's thyroiditis with papillary thyroid carcinoma. J Cancer Res Clin Oncol. 2014;140(6):1021-6.

23. Nemetz MA, Thomazelli FCS, Granero LCS, Nemetz AB, dos Santos MB. Does chronic lymphocytic thyroiditis influence the staging of differentiated thyroid carcinoma? Braz J Otorhinolaryngol. 2011;77(1):77-83.

24. Jeong JS, Kim HK, Lee CR, Park S, Park JH, Kang SW, et al. Coexistence of chronic lymphocytic thyroiditis with papillary thyroid carcinoma: clinical manifestation and prognostic outcome. J Korean Med Sci. 2012;27(8):883-9.

25. Kashima K, Yokoyama S, Noguchi S, Murakami N, Yamashita H, Watanabe $\mathrm{S}$, et al. Chronic thyroiditis as a favorable prognostic factor in papillary thyroid carcinoma. Thyroid. 1998;8(3):197-202.

26. Kebebew $\mathrm{E}$, Treseler PA, Ituarte $\mathrm{PH}, \mathrm{Clark} \mathrm{OH}$. Coexisting chronic lymphocytic thyroiditis and papillary thyroid cancer revisited. World J Surg. 2001;25(5):632-7

27. Yoon Y-H, Kim HJ, Lee JW, Kim JM, Koo BS. The clinicopathologic differences in papillary thyroid carcinoma with or without co-existing chronic lymphocytic thyroiditis. Eur Arch Otorhinolaryngol. 2012;269(3):1013-7.

28. Graceffa G, Patrone R, Vieni S, Campanella S, Calamia S, Laise I, et al. Association between Hashimoto's thyroiditis and papillary thyroid carcinoma: a retrospective analysis of 305 patients. BMC Endocr Disord. 2019;19(1):1-6.

29. Selek A, Cetinarslan B, Tarkun I, Canturk Z, Ustuner B, Akyay Z. Thyroid autoimmunity: is really associated with papillary thyroid carcinoma? Eur Arch Otorhinolaryngol. 2017:274(3):1677-81.

30. Topaloglu O, Baser H, Cuhaci FN, Sungu N, Yalcin A, Ersoy R, et al. Malignancy is associated with microcalcification and higher AP/T ratio in ultrasonography, but not with Hashimoto's thyroiditis in histopathology in patients with thyroid nodules evaluated as Bethesda category III (AUS) FLUS) in cytology. Endocrine. 2016;54(1):156-68.

31. Zeng R, Zhao M, Niu H, Yang K, Shou T, Zhang G, et al. Relationship between Hashimoto's thyroiditis and papillary thyroid carcinoma in children and adolescents. Eur Rev Med Pharmacol Sci. 2018;22:7778-87.

32. Mohamed SY, Ibrahim TR, Elbasateeny SS, Abdelaziz LA, Farouk S, Yassin MA, et al. Clinicopathological characterization and prognostic implication 
of FOXP3 and CK19 expression in papillary thyroid carcinoma and concomitant Hashimoto's thyroiditis. Sci Rep. 2020;10(1):10651.

33. Thyroiditis CL. Association between nonspecific chronic lymphocytic Thyroiditis and differentiated epithelial thyroid carcinoma: Clinicopathological analysis of patients who underwent thyroidectomy in a tertiary Care Center in Sri Lanka. World. 2018;10(2):119-26.

34. Repplinger D, Bargren A, Zhang Y-W, Adler JT, Haymart M, Chen H. Is Hashimoto's thyroiditis a risk factor for papillary thyroid cancer? J Surg Res. 2008;150(1):49-52.

35. Paparodis R, Imam S, Todorova-Koteva K, Staii A, Jaume JC. Hashimoto's thyroiditis pathology and risk for thyroid cancer. Thyroid. 2014;24(7):1107-14.

36. Anil C, Goksel S, Gursoy A. Hashimoto's thyroiditis is not associated with increased risk of thyroid cancer in patients with thyroid nodules: a singlecenter prospective study. Thyroid. 2010;20(6):601-6.

37. Konturek A, Barczyński M, Wierzchowski W, Stopa M, Nowak W. Coexistence of papillary thyroid cancer with Hashimoto thyroiditis. Langenbeck's Arch Surg. 2013;398(3):389-94.

38. Mukasa K, Noh JY, Kunii Y, Matsumoto M, Sato S, Yasuda S, et al. Prevalence of malignant tumors and adenomatous lesions detected by ultrasonographic screening in patients with autoimmune thyroid diseases. Thyroid. 2011;21(1):37-41.

39. Mateša-Anić $D$, Mateša $N$, Dabelić $N$, Kusić Z. Coexistence of papillary carcinoma and Hashimoto's thyroiditis. Acta Clin Croatica. 2009;48(1):9-12.

40. Larson SD, Jackson LN, Riall TS, Uchida T, Thomas RP, Qiu S, et al. Increased incidence of well-differentiated thyroid cancer associated with Hashimoto thyroiditis and the role of the PI3K/Akt pathway. J Am Coll Surg. 2007;204(5):764-73.

41. Zayed AA, Ali MKM, Jaber OI, Moh'd JS, Ashhab AA, Al_Shweiat WM, et al. Is Hashimoto's thyroiditis a risk factor for medullary thyroid carcinoma? Our experience and a literature review. Endocrine. 2015;48(2):629-36.

42. Gul K, Dirikoc A, Kiyak G, Ersoy PE, Ugras NS, Ersoy R, et al. The association between thyroid carcinoma and Hashimoto's thyroiditis: the ultrasonographic and histopathologic characteristics of malignant nodules. Thyroid. 2010;20(8):873-8.

43. Mazokopakis EE, Tzortzinis AA, Dalieraki-Ott El, Tsartsalis AN, Syros PK, Karefilakis CM, et al. Coexistence of Hashimoto's thyroiditis with papillary thyroid carcinoma. A retrospective study. Hormones. 2010;9(4):312-7.

44. Sclafani AP, Valdes M, Cho H. Hashimoto's thyroiditis and carcinoma of the thyroid: optimal management. Laryngoscope. 1993;103(8):845-9.

45. Peterson CA, Shidler FP. Lymphocytic thyroiditis in 757 thyroid operations. Am J Surg. 1957;94(2):223-31.

46. Zhang Y, Ma X-P, Deng F-S, Liu Z-R, Wei H-Q, Wang X-H, et al. The effect of chronic lymphocytic thyroiditis on patients with thyroid cancer. World J Surg Oncol. 2014;12(1):277.

47. Holm L-E, Blomgren H, Löwhagen T. Cancer risks in patients with chronic lymphocytic thyroiditis. N Engl J Med. 1985;312(10):601-4.

48. Silva de Morais N, Stuart J, Guan H, Wang Z, Cibas ES, Frates MC, et al. The impact of Hashimoto Thyroiditis on thyroid nodule cytology and risk of thyroid Cancer. Journal of the endocrine. Society. 2019;3(4):791-800.

49. Jackson D, Handelsman RS, Farrá JC, Lew JI. Increased incidental thyroid Cancer in patients with subclinical chronic lymphocytic Thyroiditis. J Surg Res. 2020;245:115-8.

50. Keskin M, Savas-Erdeve S, Aycan Z. Co-existence of thyroid nodule and thyroid Cancer in children and adolescents with Hashimoto Thyroiditis: a single-center study. Horm Res Paediatr. 2016;85(3):181-7.

51. Liu Y, Li C, Zhao W, Wang Y. Hashimoto's Thyroiditis is an important risk factor of papillary thyroid microcarcinoma in younger adults. Horm Metab Res. 2017;49(10):732-8.

52. Radetti G, Loche S, D'Antonio V, Salerno M, Guzzetti C, Aversa T, et al. Influence of Hashimoto Thyroiditis on the development of thyroid nodules and Cancer in children and adolescents. J Endocrine Soc. 2019;3(3):607-16.

53. Won JH, Lee JY, Hong $\mathrm{HS}$, Jeong $\mathrm{SH}$. Thyroid nodules and cancer in children and adolescents affected by Hashimoto's thyroiditis. Br J Radiol. 2018;91(1087):20180014.

54. Boi F, Pani F, Calò PG, Lai ML, Mariotti S. High prevalence of papillary thyroid carcinoma in nodular Hashimoto's thyroiditis at the first diagnosis and during the follow-up. J Endocrinol Investig. 2018;41(4):395-402.

55. Gabalec F, Srbova L, Nova M, Hovorkova E, Hornychova H, Jakubikova I, et al. Impact of Hashimoto's thyroiditis, TSH levels, and anti-thyroid antibody positivity on differentiated thyroid carcinoma incidence. Endokrynol Pol. 2016;67(1):48-53.

56. Büyükaşık O, Hasdemir AO, Yalçın E, Celep B, Sengül S, Yandakçı K, et al. The association between thyroid malignancy and chronic lymphocytic thyroiditis: should it alter the surgical approach? Endokrynol Pol. 2011;62(4):303-8

57. Chen $\mathrm{YK}$, Lin $\mathrm{CL}$, Cheng FT, Sung $\mathrm{FC}$, Kao CH. Cancer risk in patients with Hashimoto's thyroiditis: a nationwide cohort study. Br J Cancer. 2013;109(9):2496-501.

58. Uhliarova B, Hajtman A. Hashimoto's thyroiditis-an independent risk factor for papillary carcinoma. Bra J Otorhinolaryngol. 2018;84(6):729-35.

59. Mincer DL, Jialal I. Hashimoto Thyroiditis. [Updated 2020 Aug 10]. In: StatPearls [Internet]. Treasure Island (FL): StatPearls Publishing; 2021. Available from: https://www.ncbi.nlm.nih.gov/books/NBK459262/.

60. Hussein O, Abdelwahab K, Hamdy O, Awny S, Megahed NA, Hafez MT, et al. Thyroid cancer associated with Hashimoto thyroiditis: similarities and differences in an endemic area. J Egypt Natl Cancer Inst. 2020;32(1):1-4

61. Anand A, Singh KR, Kushwaha JK, Hussain N, Sonkar AA. Papillary thyroid cancer and Hashimoto's thyroiditis: an association less understood. Indian J Surg Oncol. 2014;5(3):199-204.

62. Kadam SM, Ghadhban BR, AbduljabbarSaleh M. Hashimoto's Thyroiditis increases risk for differentiated thyroid carcinoma. Ann Romanian Soc Cell Biol. 2021;25(6):5952-61.

63. Haugen BR, Alexander EK, Bible KC, Doherty GM, Mandel SJ, Nikiforov YE, et al. 2015 American Thyroid Association management guidelines for adult patients with thyroid nodules and differentiated thyroid cancer: the American Thyroid Association guidelines task force on thyroid nodules and differentiated thyroid cancer. Thyroid. 2016;26(1):1-133.

64. Feldt-Rasmussen U. Hashimoto's thyroiditis as a risk factor for thyroid cancer. Curr Opin Endocrinol Diabetes Obesity. 2020;27(5):364-71.

65. Viola D, Elisei R. Management of Medullary Thyroid Cancer. Endocrinol Metab Clin. 2019;48(1):285-301.

66. Stamatakos M, Paraskeva P, Stefanaki C, Katsaronis P, Lazaris A, Safioleas $\mathrm{K}$, et al. Medullary thyroid carcinoma: the third most common thyroid cancer reviewed. Oncol Lett. 2011;2(1):49-53.

67. Zeman V, Hanika J. Bilateral pheochromocytoma of the adrenal glands and a solitary neurofibroma of the dorsal mucosa of the tongue associated with a solid medullary carcinoma of the thyroid gland in Hashimoto's lymphocytic thyroiditis. Cesk Patol. 1977;13(4):185-91.

68. Weiss LM, Weinberg DS, Warhol MJ. Medullary carcinoma arising in a thyroid with Hashimoto's disease. Am J Clin Pathol. 1983;80(4):534-8.

69. De Pasquale L, Sommaruga L, Andreani S, Bastagli A. Hashimoto's thyroiditis and medullary carcinoma in the same gland: a purely random occurrence? Chir Ital. 2004;56(4):557-62.

70. Segal K, Ben-Bassat M, Avraham A, Har-El G, Sidi J. Hashimoto's thyroiditis and carcinoma of the thyroid gland. Int Surg. 1985;70(3):205-9.

71. Gaskin D, Parai SK, Parai MR. Hashimoto's thyroiditis with medullary carcinoma. Can J Surg. 1992;35(5):528-30.

72. Mousa U, Gursoy A, Ozdemir H, Moray G. Medullary thyroid carcinoma in a patient with Hashimoto's thyroiditis diagnosed by calcitonin washout from a thyroid nodule. Diagn Cytopathol. 2013;41(7):644-6.

\section{Publisher's Note}

Springer Nature remains neutral with regard to jurisdictional claims in published maps and institutional affiliations. 